\section{PM0-163 FIBROSCAN DELINEATES SIGNIFICANT FIBROSIS BETTER THAN THE KING'S FIBROSIS SCORE, APRI AND FIB4 SCORE WHEN CORRELATED TO LIVER BIOPSY IN A LARGE MONOCENTRIC COHORT OF HEPATITIS C PATIENTS}

doi:10.1136/gutjnl-2012-302514b.163

L-M See, ${ }^{*}$ B Kok, N Kamparidis, M-A Badot, S Hodgson, I Carey, D Joshi, M Heneghan, P Harrison, K Agarwal. Institute of Liver Studies, King's College Hospital, London, UK

Introduction In the context of consideration of antiviral therapy for hepatitis $\mathrm{C}$ virus (HCV), fibrosis assessment is critical. An increasing array of non-invasive blood panels are in use; in addition Liver Stiffness Measurements (LSM) by Fibroscan is an established clinical tool in some clinical centres. Liver biopsy is however still perceived as the "silver" standard for fibrosis assessment in HCV. The aim of our study was to evaluate the diagnostic accuracy of LSM, King's Fibrosis Score (KFS), APRI (aspartate aminotransferase to Platelet Ratio) and FIB4 score (age, platelets, aspartate aminotransferase and alanine aminotransferase) in predicting significant fibrosis (Ishak score F3-F6) and cirrhosis (F5-F6) in a large monocentric cohort of HCV patients.

Methods Retrospective data collection was performed on 484 patients with hepatitis $\mathrm{C}$ that underwent both Fibroscan and liver biopsy at King's College Hospital between November 2006 and November 2011. Data were analysed to correlate liver biopsy results with LSM, KFS, APRI and FIB4 score. Each biopsy was assessed via Ishak score with three specialist Liver Histopathologists. All Fibroscans were performed by two trained operators. Each test was correlated against liver biopsy findings using ROC curves, sensitivity and specificity.

Results For predicting significant fibrosis (F3-F6), the area under receiver operating curves $95 \%$ CIs were 0.81 ( 0.77 to $0.85, \mathrm{p}<0.001$ ) for LSM; 0.71 ( 0.66 to $0.76, \mathrm{p}<0.001$ ) for KFS; 0.71 (0.66 to 0.76 , $\mathrm{p}<0.001)$ for APRI and $0.79(0.73$ to $0.84, \mathrm{p}<0.001)$ for FIB4 score. In the diagnosis of cirrhosis, the area under receiver operating curves was 0.83 (0.76 to $0.9, \mathrm{p}<0.001)$ for LSM, 0.72 (0.64 to $0.79, \mathrm{p}<0.001)$ for KFS, 0.67 ( 0.59 to $0.75, \mathrm{p}<0.001)$ for APRI and 0.77 (0.68 to 0.85 , $\mathrm{p}<0.001)$ for FIB4 score respectively. A LSM threshold of $7.8 \mathrm{kPa}$ had a $68 \%$ sensitivity and $81 \%$ specificity to detect significant fibrosis and a threshold of $10.25 \mathrm{kPa}$ had a sensitivity of $75 \%$ and a specificity of $80 \%$ in detecting cirrhosis.

Conclusion In our real-life monocentric HCV population, liver stiffness measurement via Fibroscan performed best in prediction of both moderate fibrosis and cirrhosis in comparison to the KFS, APRI and FIB4. However, all these panels performed reasonably in delineating significant fibrosis and cirrhosis. An increased availability of Fibroscan, coupled with non-invasive fibrosis panels in primary or outreach settings can radically improve the clinical assessment and evaluation of HCV patients for HCV therapy, while also defraying cost and improving safety and acceptability from a patient perspective. Non-invasive fibrosis assessment needs to be widely available.

Competing interests None declared.

\section{PMO-164 HCV P22 ANTIGEN TEST: SEROLOGICAL RESPONSE AND DIAGNOSTIC ADVANTAGES}

doi:10.1136/gutjnl-2012-302514b.164

L Lee, * G Perrett, P Howard, D Makanjuola, J Clark. Epsom and St Helier University Hospitals NHS Trust, London, UK

Introduction The p22 ELISA stains for part of the nucleocapside of hepatitis $\mathrm{C}$ virus (HCV) and has recently become commercially available. The current British Society of Gastroenterology guidelines recommend ELISA towards antibodies against HCV Ab. Only $27 \%$ of patients with acute HCV are able to clear the virus, ${ }^{1}$ however, early treatment in the acute phase is known to improve outcomes and rates of Sustained Virological Response. ${ }^{2}$ This has been advocated in certain subgroups (patients co-infected with HIV or moderate-severe liver disease). This case demonstrates the serological response during an acute hepatitis infection with regards to ALT, HCV Ab, HCV RNA and the new p22 HCV antigen test.

Methods Sequential blood samples were obtained from a patient with end stage renal failure who developed acute HCV infection while on haemodialysis. Liver function tests, ELISA to antibody to HCV, HCV RNA PCR and antigens to p22 were performed retrospectively.

Results The p22 ELISA result became positive at an earlier time point before a rise in ALT or the ELISA HCV antibody result.

Conclusion The p22 ELISA is a robust and reliable test that allows for earlier detection of HCV viraemia. This would be of crucial importance in patient subgroups that need early treatment in the acute phase or in populations where early detection would be required to reduce risk of cross-transmission (eg, haemodialysis units). This test offers several advantages over PCR, which though sensitive, involves complex methodology that makes it unsuitable for diagnostic laboratories. Further investigation would be required to establish the role of treating during the hyper-acute phase, in patients with negative HCV antibody and normal ALT.

Abstract PM0-164 Table 1

\begin{tabular}{lllll}
\hline Week & ALT (IU/I) & HCV Ab result & HCV antigen result & HCV RNA PCR (IU/mI) \\
\hline 0 & 26 & $-v e$ & $-v e$ & $-v e$ \\
2 & 22 & $-v e$ & $+v e(3474)$ & 140000 \\
8 & 394 & $-v e$ & $+v e(>20000)$ & $2.1 \mathrm{~m}$ \\
11 & 474 & $+v e$ & $+v e(2399)$ & 106000 \\
16 & 181 & $+v e$ & $+v e(6573)$ & 558000 \\
\hline
\end{tabular}

Competing interests None declared.

\section{REFERENCES}

1. Micallef JM, Kaldor JM, Dore GJ. Spontaneous viral clearance following acute hepatitis C infection: a systematic review of longitudinal studies. J Viral Hepat 2006; 13:34-41.

2. Corey KE, Mendez-Navarro J, Gorospe EC, et al. Early treatment improves outcomes in acute hepatitis C virus infection: a meta-analysis. J Viral Hepat 2010;17:201-7.

\section{PM0-165 POTENTIAL IMPACT OF PROTEASE INHIBITORS IN THE SOUTH WEST PENINSULA HEPATITIS C POPULATION}

doi:10.1136/gutjnl-2012-302514b.165

${ }^{1} \mathrm{M}$ Saunders, ${ }^{*}{ }^{2} \mathrm{C}$ Sieberhagen, ${ }^{2} \mathrm{~L}$ Taylor, ${ }^{1} \mathrm{~F}$ Fry, ${ }^{3} \mathrm{M}$ McKenna, ${ }^{4} \mathrm{~S}$ Needs, ${ }^{5} \mathrm{R}$ Chimakurthi, ${ }^{2} \mathrm{M}$ Cramp. ${ }^{1}$ Royal Devon and Exeter Hospital, Exeter, UK; ${ }^{2}$ Hepatology Department, Derriford Hospital, Plymouth, UK; ${ }^{3}$ Treliske Hospital, Truro, UK; ${ }^{4}$ Torbay Hospital, Torbay, UK; ${ }^{5}$ North Devon District Hospital, Barnstaple, UK

Introduction Treatment of hepatitis $\mathrm{C}$ virus (HCV) with pegylated interferon and ribavirin achieves a sustained viral response (SVR) in $40 \%-50 \%$ of genotype 1 patients. ${ }^{1}$ Protease inhibitors (PIs) increase SVR rates in treatment naïve genotype 1 patients to $63 \%-75 \%$, but it is in those who failed to achieve an SVR with previous treatment that the most significant differences have been observed, particularly in those who relapse. ${ }^{1}$ This study assessed the potential beneficial impact of PIs in a real world population undergoing HCV treatment in the South West Peninsula, identifying the number of eligible patients and considering population-specific issues to treatment.

Methods All patients treated for HCV in the South West Peninsula between January 2008 and December 2010 were identified by HCV 